0.001, chronic: $\mathrm{r}=0.556, \mathrm{p}<0.001)$ and preparedness to perform practical procedures (acute: $\mathrm{r}=0.724, \mathrm{p}<0.001$, chronic: $\mathrm{r}=0.433$, $\mathrm{p}<0.001)$.

Confidence prescribing mental health drugs was lower than all other comparison items (simple analgesia: $r=0.854$, bronchodilators: $r=0.789$, antimicrobials: $r=0.772$, inhaled steroids: $r=$ 0.720 , intravenous fluids: $r=0.702$, oral anti-diabetics: $r=0.611$, anticoagulants: $r=0.515$, narcotics: $r=0.514$, insulin: $r=0.206$; $\mathrm{p}<0.001)$

Conclusion. These results identify a disparity in foundation doctors' self-reported preparedness to treat acute and chronic mental health conditions and prescribe psychotropic medications, compared to a variety of physical health domains. To our knowledge this is the first large-scale study to empirically test a potential discrepancy between newly-qualified doctors' preparedness to treat patients' mental and physical health needs. Medical school education and foundation training may therefore present a fruitful opportunity to improve care for patients with psychiatric conditions within general hospital settings.

\section{A pilot project to increase trainee engagement using a social media platform - outcomes and lessons learnt}

Amy Grimason ${ }^{1 \star}$, Shevonne Matheiken ${ }^{2}$, Laura Somerville ${ }^{3}$, Fiona Martin ${ }^{4}$, Luke Baker ${ }^{5}$, Kabir Garg ${ }^{6}$, Aastha Sharma ${ }^{7}$ and Simon George Morris ${ }^{8}$

${ }^{1}$ Northern Ireland Medical and Dental Training Agency, Belfast Health and Social Care Trust; ${ }^{2}$ Northamtonshire Healthcare NHS Foundation Trust; ${ }^{3}$ Northern Ireland Medical and Dental Training Agency, South Eastern Health and Social Care Trust; ${ }^{4}$ Northern Ireland Medical and Dental Training Agency, Northern Health and Social Care Trust; ${ }^{5}$ South London and Maudsley NHS Foundation Trust, Institute of Naval Medicine; ${ }^{6}$ Oxleas NHS Foundation Trust, London; ${ }^{7}$ Cambridgeshire and Peterborough NHS foundation trust and ${ }^{8}$ Avon and Wiltshire Mental Health Partnership NHS Trust ${ }^{\star}$ Corresponding author.

doi: 10.1192/bjo.2021.393

Aims. Engagement with members is an important issue for the Royal College of Psychiatrists (RCPsych) and an area for ongoing development. This is an issue that extends to Psychiatry trainees and the Psychiatric Trainees' Committee (PTC) has adopted increasing engagement as one of its key aims. Divisional representatives in different areas of the UK had noted that trainees had limited knowledge of the PTC or its roles and projects both within the College and local areas. To improve this it was decided to pilot a project that established a social media platform for trainees to improve communication between the PTC, it's representatives and trainees. It was decided that Workplace (a professional version of Facebook) would be used. This had already been established in the Severn Deanery.

Method. Northern Ireland (NI) and the East of England (EoE) deaneries were selected as pilot areas for the project. Preparation for the project included collaboration with trainees from the Severn deanery and meeting with the RCPsych Digital team. A scoping questionnaire was circulated to trainees in each deanery.

Following this, two closed groups were initiated on Workplace in August 2019 for Northern Ireland and East of England trainees. Result. Results from the survey sent prior to the social media pages being established indicated there was appetite among trainees for the project. The pages were established in July 2019. The pilot project was promoted by representatives.
In the initial phases, approximately $40 \%$ of trainees signed up. Information regarding college and local events, committee meeting updates and training opportunities was disseminated on the platform. There was evidence of early use by trainees outside of the representative group.

This however was not sustained and gradually use of the platform reduced over the pilot period, both in postings and membership. A further questionnaire circulated in July 2020 highlighted trainees' concerns relating to the platform, including concerns around data protection and a high number of notifications associated with the Workplace medium. The ultimate impact on engagement was also felt to be minimal.

Conclusion. Following feedback and increasing usage costs by Workplace, it was decided not to continue with a nationwide role out of the project. COVID-19 has seen the successful use of platforms such as Microsoft Teams and these may be considered in the future, given their integration with existing trust systems.

\section{Creating a virtual, clinical scenario based teaching programme for foundation doctors in Leeds}

David Hall*, Thomas Lane and Alexander Harbinson

Leeds and York Partnership NHS Foundation Trust

${ }^{*}$ Corresponding author.

doi: 10.1192/bjo.2021.394

Aims. Through consultation with foundation doctors on their psychiatry placements in Leeds, we established that they didn't feel the trustwide teaching programme met their needs, with them rating the relevance as 5.8 on a $1-10$ Likert scale. They also reported their access to formal and informal teaching had been limited by COVID-19 restrictions. We aimed to create an accessible teaching programme that met their developmental needs as set out by the Foundation curriculum, as well as their confidence and skill set in dealing with common mental health presentations. Our supplementary aims were to promote psychiatry as a career and to provide supervised teaching opportunities to core trainees.

Method. Having assessed the foundation doctors confidence in dealing with different scenarios, we created a 9 week teaching programme covering common mental health presentations they're likely to encounter during their training. The virtual sessions focus on what assessment and management skills would be expected for a foundation doctor, as well as when and how to access support and refer on. The programme was designed to be trainee led with the teaching being facilitated by core trainees as it was felt that they would best relate to the experiences and developmental needs of foundation doctors. This also provided the CTs with an opportunity to develop their teaching skills, something that has become more difficult during COVID.

Result. Through weekly feedback of the sessions we were able to demonstrate that for 8 of the 9 sessions the foundation doctors rated them as being 'useful' or 'very useful' and we're currently reviewing the topic and materials for the outlying session.

Through self-assessed ratings before and after the programme, we demonstrated significant increases in confidence in dealing with all 9 of the scenarios. All of the foundation doctors indicated that they had found the programme beneficial.

Conclusion. As shown in the results, the programme has been well received by the foundation doctors who's confidence in dealing with a range of scenarios has improved. The programme has also been well received by the trust who have asked us to repeat the it for future foundation rotations and by core trainees who were grateful of the development opportunities that this provided. 